Original article

\title{
The role of pain and disability changes after physiotherapy treatment on global perception of improvement in patients with chronic low back pain
}

\author{
Diogo André da Fonseca Pires ${ }^{a, b, *}$, Eduardo José Carvalho Brazete Cruz ${ }^{\text {b,c }}$, \\ Helena Cristina de Matos Canhão ${ }^{\text {b, d,e }}$, Carla do Rosário Delgado Nunes ${ }^{\text {a,b }}$ \\ ${ }^{a}$ NOVA National School of Public Health, Public Health Research Centre, Universidade NOVA de Lisboa, Portugal \\ ${ }^{\mathrm{b}}$ Comprehensive Health Research Center (CHRC), Portugal \\ ${ }^{c}$ Department of Physical Therapy, School of Health Care, Polytechnic Institute of Setúbal, Portugal \\ ${ }^{\mathrm{d}}$ Cedoc, EpiDoC Unit, NOVA Medical School, Nova University of Lisbon, Lisbon, Portugal \\ ${ }^{\mathrm{e}}$ Department of Rheumatology, CHULC-Hospital Curry Cabral, Lisbon, Portugal
}

\section{A R T I C L E I N F O}

\section{Keywords:}

Global perception of improvement

Chronic low back pain

Physiotherapy

Pain intensity

Disability

\begin{abstract}
A B S T R A C T
Background: The effectiveness of physiotherapy in patients with chronic low back pain is usually measured through changes in pain and disability domains. However, recent research has suggested that these two domains are not sufficient to capture all the physiotherapy benefits when patients' perspective is considered. Objective: The aim of this study was to investigate the role of pain and disability changes in explaining the global perception of improvement in patients with chronic low back pain undergoing physiotherapy. Design: Prospective cohort study.

Methods: The study was conducted on 183 patients who were referred to physiotherapy treatment due to low back pain lasting more than 12 weeks. Sociodemographic and clinical characteristics were measured at baseline, together with pain intensity and disability. Eight (post-intervention) and twelve weeks later, global perception of improvement was measured together with pain and disability. The Pearson correlation coefficient and linear regression models were used for analyses.

Results: Of the 183 participants included, 144 completed the 12-weeks follow-up. Significant and moderate correlation was found between pain and disability changes and the global perception of improvement after intervention and at the 12 -weeks follow-up. Pain and disability changes explained $20.7 \%-36.3 \%$ of the variance in the global perception of improvement.

Conclusions: Pain and disability changes are related and contributed to explaining a partial proportion of variance in the global perception of improvement. The findings suggest that these domains are not sufficient to explain and measure all of the benefits of physiotherapy when patients' global perception of improvement is considered.
\end{abstract}

\section{Introduction}

Chronic low back pain (CLBP) is one of the most prevalent health conditions worldwide (Meucci et al., 2015). In addition to the impact at the individual level, the large economic and social costs related to CLBP are well documented in the literature (Gouveia et al., 2016; March et al, 2014; Parthan et al., 2006). Subsequently, an increased research effort has been observed in order to understand the associated factors and the most effective interventions for this health condition (Wand and O'Connell, 2008). Physiotherapy modalities are recommended for patients with CLBP (Foster et al., 2018; National Institute for Health and
Care Excellence, 2016) and the evidence on this topic has accompanied a global effort of all health research.

An important part of an effective research process is the selection of appropriate outcome domains (Williamson et al., 2012). This is critical to compare and quantify the benefits (or adverse effects) associated with the applied interventions as well as to promote evidence-informed practice (Gargon et al., 2014). Physiotherapy studies on CLBP frequently measure pain and disability domains (Pires et al., 2020). A recent systematic review identified the outcome domains reported in 195 randomized controlled trials examining physiotherapy interventions for patients with CLBP (Pires et al., 2020). This review found

\footnotetext{
* Corresponding author. Centro de Investigação em Saúde Pública, Escola Nacional de Saúde Pública, Avenida Padre Cruz, Lisbon, 1600-560, Portugal.

E-mail addresses: piresdiogo.af@gmail.com, da.pires@ensp.unl.pt (D.A.F. Pires).
} 
that pain intensity and disability were used in $85 \%$ of the included studies and were the most frequent primary outcomes. Others common domains such as spine mobility and health-related quality of life were used in less than $30 \%$ of the studies (Pires et al., 2020). Therefore, pain and disability are the only domains used in a large proportion of studies.

Despite the dominance of pain and disability on outcomes assessment of patients with CLBP, preliminary evidence from qualitative studies supports that they are not sufficient when patients' perspective is considered. Hush et al. (2009) developed a qualitative study with 36 patients that recovered or did not recover from low back pain. The authors found a discrepancy between pain and disability scores and the perspective of self-reported recovery by patients suggesting the relevance of others domains (Hush et al., 2009). More recently, others studies have reinforced this view supporting a potential discord between outcome domains based on the health professional perspective and the effects of intervention that are meaningful to the patients (Gardner et al., 2015; Sanderson et al., 2010).

Consequently, patients' global perception of improvement measures have been progressively introduced into chronic pain research (Dworkin et al., 2005). These measures provide reliable information about patients' perspective on the intervention's benefits helping to interpret if changes in specific outcome domains such as pain or disability were meaningful to patients (Dworkin et al., 2008; Kamper et al., 2010b). At this point, there is a lack of quantitative studies analysing the relationship between pain and disability changes with patients' global perceptions of improvement. This knowledge may contribute to clarifying the extent to which the pain and disability domains are sufficient (or not) to analyse the effectiveness of physiotherapy considering the patients' perspective of improvement. The aim of this study was to analyse the role of pain and disability changes in explaining global perception of improvement in patients with chronic low back pain undergoing physiotherapy.

\section{Methods}

\subsection{Study design and setting}

A prospective cohort study with a 12-week follow-up was undertaken in patients seeking physiotherapy treatments for nonspecific CLBP. Between October 2015 and December 2018, potential participants were identified and recruited consecutively from 20 different outpatient clinics in Portugal. A standardized protocol was followed by local physicians and/or physiotherapists in the recruitment process. The minimum sample size required was previously established using formula $\mathrm{N}>50+8 \mathrm{~m}$ (where $\mathrm{m}$ is the number of independent variables) (Green, 1991). In this study, eight clinical and demographic variables were considered in addition to pain and disability changes. A potential loss of $20 \%$ of participants during the study was also considered. Accordingly, a minimum of 156 participants was required. All participants received oral and written information about the study and provided their informed consent prior to participating.

\subsection{Participants}

Inclusion criteria for this study were: patients aged 18 to 65 with nonspecific low back pain with at least 12 weeks duration (Airaksinen et al., 2006), with or without leg pain, pain intensity $\geq 3$ (measured by the Numeric Pain Rating Scale) on the day of the initial evaluation, and literate in Portuguese. Patients with clinical signs of serious or specific pathologies (inflammatory disorder, fracture, radicular syndrome) (Smeets et al., 2006), pregnancy, and history of back surgery or conservative treatment in the prior 12 and 3 months, respectively, were excluded. Eligibility was checked by local physiotherapists.

\subsection{Intervention}

All participants received usual care in physiotherapy, without any restriction from the research team. Although a comprehensive definition of physiotherapy treatments was provided (manual therapy; therapeutic education; therapeutic exercise; electrotherapy and physical agents), the type of treatments used and the number of sessions were the responsibility of physiotherapists. Usual care was chosen in order to reflect current practice and promote the variability of the treatments applied. Therefore, the characteristics of the intervention applied were not under analysis and it was assumed that the variability of treatments washed out specific treatment modifier effects (Kent et al., 2010). The duration of the intervention, the number of participants who did not complete the intervention and their reasons were recorded.

\subsection{Instruments}

Participants were assessed at baseline, 8 weeks after the beginning of the intervention (or earlier if they were discharged) and at the 12-week follow-up. At baseline, participants were asked to complete a sociodemographic and clinical questionnaire (see Table 1) along with the Numeric Pain Rating Scale (NPRS) and the Quebec Back Pain Disability Scale (QBPDS-PT) (Cruz et al., 2013). The Global Perceived Effect Scale (GPES-PT) (Freitas et al., 2019) was used to assess the patients' global perception of improvement. Eight and twelve weeks later, GPES-PT was applied together with NPRS and QBPDS-PT. GPES-PT is a transition scale ranging from -5 ("vastly worse") to +5 ("completely recovered"). This measure was previously translated and cross-culturally adapted in European Portuguese showing adequate convergent validity $(r=0.677)$, test-retest reliability (ICC $=0.758$ ), and responsiveness (Areas under the curve values of 0.71 and 0.83 ) (Freitas et al., 2019). To assess the average level of pain intensity, the NPRS was used. The NPRS is an 11-point self-report measure (0-10) with the labels "no pain" and "worst imaginable pain" on the ends that has proven to be valid and reliable in patients with chronic pain (Farrar et al., 2001). Functional disability was assessed using QBPDS-PT. This questionnaire consists of 20 items representing functional activities with 6 response categories each (0- "not difficult at all" to 5- "unable to do"). The total score is calculated by a summation of the scores for each individual item ranging from 0 (no disability) to 100 (severe disability). The QBPDS-PT has shown good validity $(\rho=0.62)$, test-retest reliability $($ ICC $=0.696)$ and internal

Table 1

Baseline characteristics of the participants.

\begin{tabular}{ll}
\hline Variables & Total Sample $(\mathrm{n}=183)$ \\
\hline Age $^{\mathrm{a}}$ & $48.02 \pm 10.53$ \\
BMI $\left(\mathrm{kg} / \mathrm{m}^{2}\right)^{\mathrm{a}}$ & $26.18 \pm 4.28$ \\
Gender [N (\%)] & $36(19.7 \%)$ \\
$\quad$ Male & $147(80.3 \%)$ \\
$\quad$ Female & $74(40.4 \%)$ \\
Educational level [n (\%)] & \\
$\quad$ Primary/Basic education & $109(59.6 \%)$ \\
$\quad$ High school/ & $152(83.1 \%)$ \\
$\quad$ College & $31(16.9 \%)$ \\
Working status [n (\%)] & \\
$\quad$ Employed & $58(31.7 \%)$ \\
$\quad$ Not Active & $125(68.3 \%)$ \\
Duration of pain [n (\%)] & \\
$\quad 3-24$ months & $121(66.1 \%)$ \\
$\quad>24$ months & $61(33.3 \%)$ \\
Pain Irradiation [n (\%)] & \\
$\quad$ Yes & $85(46.4 \%)$ \\
$\quad$ No & $98(53.6 \%)$ \\
Medication [n (\%)] & $5.86 \pm 1.88$ \\
$\quad$ Yes & $36.54 \pm 17.78$ \\
$\quad$ No &
\end{tabular}

a (mean $\pm \mathrm{SD}$ ). 
consistency (Cronbach's $\alpha=0.95$ ) (Cruz et al., 2013).

\subsection{Data analysis}

Descriptive statistics was used to summarize participants' characteristics at baseline. To compare the characteristics between participants who completed and did not completed the study, the chi-square test or Mann-Whitney $U$-test were used. All variables were assessed for normality and outliers. Absolute change in pain and disability were computed by subtracting baseline scores from post-intervention and 12 weeks follow-up scores. Thus, positive changes indicated improvement, while negative changes indicated worsening. Based on absolute changes and baseline scores, the percentage change was calculated. Pearson correlation coefficient was performed to quantify the association between the GPES scores and pain and disability changes. The correlation coefficients were interpreted as follows: $r<0.10$, no association; $r=$ $0.10-0.39$, weak; $r=0.40-0.69$, moderate; $r=0.70-0.89$, strong; and $r$ $=0.90-1.0$, very strong (Schober and Schwarte, 2018).

Linear regression models were preformed to investigate the association of changes in pain and disability (independent variables) in relation to the GPES scores (dependent variable). Previously, a univariate linear regression was conducted between each baseline variable (clinical and sociodemographic characteristics) and the dependent variable. Baseline variables with a $p$ value $\leq 0.2$ were then entered in all multivariate regression models as covariates. After that, multivariate models were performed (method: forward stepwise) according to a predefined sequence of steps. First, the absolute changes in pain were entered alone into the regression equation. Second, the same was performed for the absolute changes in disability (without the presence of absolute changes in pain). Third, the absolute changes in pain and disability were entered together into the regression equation. To quantify the variance in the dependent variable (GPES) attributable to the pain and disability variance, the $\mathrm{R}^{2}$ was recorded in each step of the analysis. In addition, the relative importance of predictors was used to understand the contribution of each independent variable in the regression equation (Tonidandel and LeBreton, 2011). The same analysis was repeated for the percentage changes after the intervention and at the 12-week follow-up. Variance inflation factors (VIF) were used to check multicollinearity. VIFs greater than 10 were considered indicative of serious multicollinearity problems. Data analysis was performed using SPSS (version 24.0; IBM, Chicago, IL). A significance level of 0.05 was chosen for this study.

\section{Results}

\subsection{Participants}

Of the 235 potential participants identified, 183 participants with CLBP were considered eligible and accepted to participate in this study. Of those, 173 (94.5\%) completed the physiotherapy treatment and 144 (78.7\%) completed the follow-up at 12 weeks. A study flowchart and reasons for dropouts are described in Fig. 1. Table 1 describes the characteristics of all participants assessed at baseline. Participants who failed follow-up evaluations differ from those that did not fail in pain

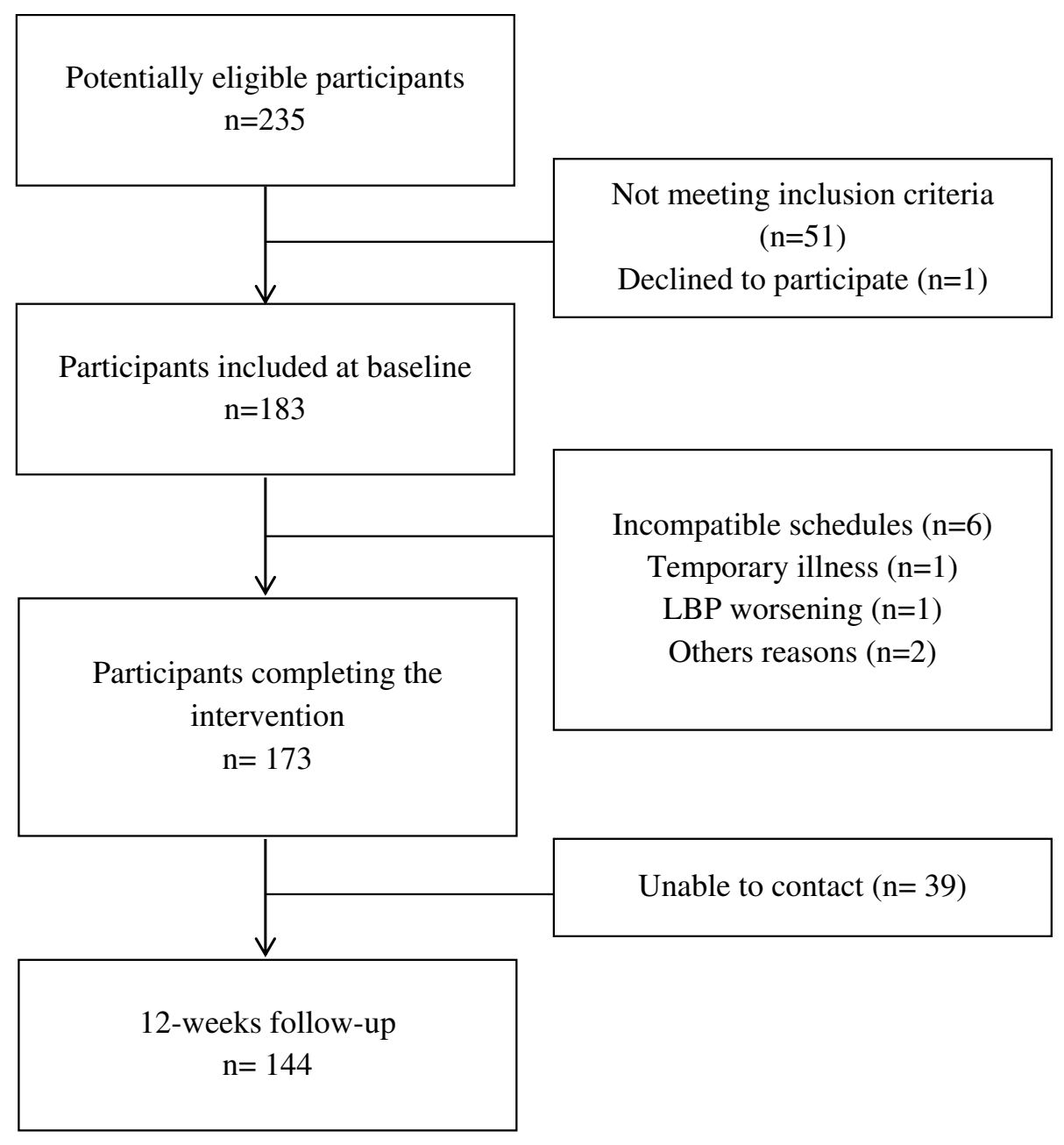

Fig. 1. Study flow-chart. 
Table 2

Mean scores of GPES and changes from baseline in pain intensity and disability.

\begin{tabular}{|c|c|c|c|c|c|}
\hline & \multirow[t]{2}{*}{ GPES Scores } & \multicolumn{2}{|c|}{ Absolute Changes } & \multicolumn{2}{|c|}{ Percentage Changes } \\
\hline & & Pain & Disability & Pain & Disability \\
\hline Post-intervention & $3.02 \pm 1.30$ & $2.56 \pm 2.48$ & $13.63 \pm 16.90$ & $41.73 \pm 45.82$ & $36.03 \pm 38.99$ \\
\hline 12-Weeks follow-up & $2.71 \pm 1.74$ & $2.04 \pm 2.58$ & $10.68 \pm 17.41$ & $33.72 \pm 46.91$ & $24.87 \pm 50.69$ \\
\hline
\end{tabular}

Table 3

Correlation between GPES scores and changes in pain and disability.

\begin{tabular}{lllllll}
\hline & \multicolumn{2}{l}{ Absolute Changes } & & \multicolumn{2}{c}{ Percentage Changes } \\
\cline { 2 - 3 } & Pain & Disability & & Pain & Disability \\
\hline Post-intervention GPES scores & 0.44 & 0.51 & & 0.48 & 0.55 \\
12-Weeks follow-up GPES scores & 0.27 & 0.41 & & 0.28 & 0.39 \\
\hline
\end{tabular}

*All correlations were significant $(\mathrm{p}<0.01)$.

intensity $(\mathrm{p}=0.009)$, irradiated pain $(\mathrm{p}=0.042)$ and medication use ( $\mathrm{p}$ $=0.033$ ). Significant differences in other variables evaluated at the baseline were not found. The mean scores for changes in pain and disability measures as well as the GPES mean scores after the intervention and at the 12-week follow up are presented in Table 2.

\subsection{Correlations analysis}

Pearson correlations between the GPES scores and improvements in pain and disability are outlined in Table 3 . All correlations were statistically significant $(\mathrm{p}<0.001$ ), weak to moderate ( $\mathrm{r}$ ranging from 0.27 to $0.55)$ and positive.

\subsection{Linear regression analysis}

The explained variance for the GPES scores in the full regression model ranged between $14.4 \%$ and $37.4 \%$ (Tables 4 and 5). A maximal explained variance was obtained when percentage changes in pain and disability were analysed together. Partial regression models with pain or disability separately, suggest a greater contribution of disability changes to the GPES when compared to pain intensity improvement (Tables 4 and 5). The same results were observed for the relative importance values. Regression models after the intervention and including change scores in percentage obtained higher values of variance. The VIFs were

\section{Table 4}

Linear regression results examining contributions of post-intervention changes in pain and disability to GPES scores.

\begin{tabular}{|c|c|c|c|c|}
\hline & $\begin{array}{l}\text { Independent Variables } \\
\text { entered in the model }\end{array}$ & $\begin{array}{l}\text { Adjust } \\
\mathrm{R}^{2}\end{array}$ & $\begin{array}{l}\text { Predictors relative } \\
\text { importance }\end{array}$ & $p$ \\
\hline \multicolumn{5}{|c|}{ Using Absolute Changes } \\
\hline \multirow{2}{*}{$\begin{array}{l}\text { 1st } \\
\text { step }\end{array}$} & $\Delta$ Pain & 0.194 & 1.0 & 0.001 \\
\hline & $\Delta$ Pain $^{\mathrm{a}}$ & 0.219 & 0.88 & 0.001 \\
\hline \multirow{2}{*}{$\begin{array}{l}\text { 2nd } \\
\text { step }\end{array}$} & $\Delta$ Disability & 0.253 & 1.0 & 0.001 \\
\hline & $\Delta$ Disability $^{\mathrm{a}}$ & 0.264 & 0.94 & 0.001 \\
\hline \multirow{4}{*}{$\begin{array}{l}\text { 3rd } \\
\text { step }\end{array}$} & $\Delta$ Pain & 0.306 & 0.33 & 0.001 \\
\hline & $\Delta$ Disability & & 0.67 & 0.001 \\
\hline & $\Delta$ Pain & 0.322 & 0.33 & 0.001 \\
\hline & $\Delta$ Disability $^{\mathrm{a}}$ & & 0.57 & 0.001 \\
\hline \multicolumn{5}{|c|}{ Using Percentage Changes } \\
\hline \multirow{2}{*}{$\begin{array}{l}\text { 1st } \\
\text { step }\end{array}$} & $\Delta$ Pain & 0.225 & 1.0 & 0.001 \\
\hline & $\Delta$ Pain $^{\mathrm{a}}$ & 0.245 & 0.90 & 0.001 \\
\hline \multirow{2}{*}{$\begin{array}{l}\text { 2nd } \\
\text { step }\end{array}$} & $\Delta$ Disability & 0.293 & 1.0 & 0.001 \\
\hline & $\Delta$ Disability $^{\mathrm{a}}$ & 0.301 & 0.96 & 0.001 \\
\hline \multirow{4}{*}{$\begin{array}{l}\text { 3rd } \\
\text { step }\end{array}$} & $\Delta$ Pain & 0.363 & 0.34 & 0.001 \\
\hline & $\Delta$ Disability & & 0.66 & 0.001 \\
\hline & $\Delta$ Pain & 0.374 & 0.34 & 0.001 \\
\hline & $\Delta$ Disability $^{\mathrm{a}}$ & & 0.59 & 0.001 \\
\hline
\end{tabular}

a Adjusted to baseline variables (BMI; working status; educational level).
Table 5

Linear regression results examining contributions of 12-Weeks follow-up changes in pain and disability to GPES scores.

\begin{tabular}{|c|c|c|c|c|}
\hline & $\begin{array}{l}\text { Independent Variables } \\
\text { entered in the model }\end{array}$ & $\begin{array}{l}\text { Adjust } \\
\mathrm{R}^{2}\end{array}$ & $\begin{array}{l}\text { Predictors relative } \\
\text { importance }\end{array}$ & $p$ \\
\hline \multicolumn{5}{|c|}{ Using Absolute Changes } \\
\hline \multirow{2}{*}{ 1st } & $\Delta$ Pain & 0.144 & 1.0 & 0.001 \\
\hline & $\Delta$ Pain $^{\mathrm{a}}$ & 0.145 & 0.95 & 0.001 \\
\hline \multirow{2}{*}{$\begin{array}{l}\text { 2nd } \\
\text { step }\end{array}$} & $\Delta$ Disability & 0.179 & 1.0 & 0.001 \\
\hline & $\Delta$ Disability $^{\mathrm{a}}$ & 0.179 & 0.97 & 0.001 \\
\hline \multirow{4}{*}{$\begin{array}{l}\text { 3rd } \\
\text { step }\end{array}$} & $\Delta$ Pain & 0.207 & 0.33 & 0.015 \\
\hline & $\Delta$ Disability & & 0.67 & 0.001 \\
\hline & $\Delta$ Pain & 0.207 & 0.33 & 0.015 \\
\hline & $\Delta$ Disability $^{\mathrm{a}}$ & & 0.67 & 0.001 \\
\hline \multicolumn{5}{|c|}{ Using Percentage Changes } \\
\hline \multirow{2}{*}{ 1st } & $\Delta$ Pain & 0.160 & 1.0 & 0.001 \\
\hline & $\Delta$ Pain $^{\mathrm{a}}$ & 0.160 & 1.0 & 0.001 \\
\hline \multirow{2}{*}{$\begin{array}{l}\text { 2nd } \\
\text { step }\end{array}$} & $\Delta$ Disability & 0.184 & 1.0 & 0.001 \\
\hline & $\Delta$ Disability ${ }^{\mathrm{a}}$ & 0.184 & 1.0 & 0.001 \\
\hline \multirow{4}{*}{$\begin{array}{l}\text { 3rd } \\
\text { step }\end{array}$} & $\Delta$ Pain & 0.222 & 0.39 & 0.006 \\
\hline & $\Delta$ Disability & & 0.61 & 0.001 \\
\hline & $\Delta$ Pain & 0.222 & 0.39 & 0.006 \\
\hline & $\Delta$ Disability $^{\mathrm{a}}$ & & 0.61 & 0.001 \\
\hline
\end{tabular}

a Adjusted to baseline variables (Age).

less than 2 in all models indicating an absence of multicollinearity problems.

\section{Discussion}

In the present study, the role of pain and disability changes in accounting for the global perception of improvement in patients with CLBP undergoing physiotherapy was analysed. The main findings are that the pain intensity and disability changes during the intervention demonstrated a modest contribution to the GPES scores. Despite the independent and significant associations, pain intensity and disability changes accounted for a small proportion of total GPES variance even when considered together. In addition, correlations between pain and disability changes with the GPES scores were mostly close to or less than 0.5 . These data suggest that these domains represent different constructs and greater changes in pain and disability may not necessarily mean higher levels of perceived improvement.

Changes in pain and disability explained up to $36.3 \%$ of the variance in the GPES scores in this study. Although a complete accounting of the global perception of improvement is not expected, a large proportion of variance remains unexplained. The role of other benefits of interventions, not assessed in this study, may help to explain these findings. Previous studies that included chronic pain patients have demonstrated significant associations of changes in other domains such as fatigue (Hudson et al., 2009), sleep (Geisser et al., 2010), work (Hudson et al., 2009), depression (Geisser et al., 2010; Scott and McCracken, 2015) or social function (Scott and McCracken, 2015) with the global perception of improvement. Scott and McCracken (2015) reported that perceived changes in pain, mood and physical, social, and work-related activities explained 64\% of the variance in Patient Global Impression of Change. These values of variance are substantially higher than those found in this study. Qualitative studies have also reported similar findings. Hush et al. (2009) reported that perception of recovery 
is mediated by patients' appraisal of their function and pain intensity but they are not a reliable indicator of recovery. Accordingly, other authors have suggested that global perception of improvement may incorporate a variety of other domains such as self-efficacy, self-esteem, spontaneity or "feeling positive emotions" (Evans et al., 2014; Walton, 2013). This set of domains fits in the mental health area and can be particularly important in patients' adaptation and readjustment to the health condition. This point of view and the way these intervention benefits seem to contribute to the perception of improvement have been described in previous studies (Beaton et al., 2001; Walton, 2013). Therefore, there appears to be reason to anticipate that the evaluation of other variables potentially modifiable by physiotherapy interventions could contribute to a better understanding of the GPES scores.

Pain is the most common symptom of CLBP and an important baseline predictor for the intervention success (Cecchi et al., 2014; Verkerk et al., 2013). Interestingly, a greater association and contribution of disability changes to GPES scores in relation to pain intensity changes was consistently identified in this study. The highest relative importance values for disability changes (ranging from 0.61 to 0.67 ) compared to those observed for changes in pain intensity (ranging from 0.33 to 0.39 ) also supports this assumption. Several reasons for these findings can be discussed. Firstly, chronic pain patients may not expect a complete resolution of their pain condition (Evans et al., 2014). Thus, the importance attributed by patients to improvements in other variables such as disability may be greater than improvements in pain. The secondary role of pain in patients with CLBP was also demonstrated in the study conducted by Kamper et al. (2010a). They found that pain improvements accurately identified patients with acute low back pain who perceive a complete recovery. However, the odd ratios values representing this relationship were substantially lower in patients with CLBP (Kamper et al., 2010a). Secondly, recent studies have demonstrated early pain changes in the intervention and its predictive value for success in others variables after the intervention (Cook et al., 2017; Mansell et al., 2017). Therefore, pain changes may act as facilitators for the disability changes that are more easily perceived as important by the patients after the intervention. Finally, the type of instrument used to measure pain (unidimensional scale) and disability (multi-item scale) may not be irrelevant to the observed results. Considering the complexity of pain experience, limited information can be captured using a single and unidimensional measure such as the NPRS. In contrast, the QBPDS includes a variety of functional activities more easily understood by the patients and representatives of their daily restrictions due to pain. This may also help to explain why disability is better related to global perception of improvement.

In addition to the reasons described above, the role of the intervention adopted in this study should not be underestimated. The influence of the type of intervention and its goals in the domains with the greatest contribution to the global perception of improvement scales has been reported. Geisser et al. (2010) found that pain changes were the main contributor when fibromyalgia patients assessed the perceived benefits with pharmacological treatment. In contrast, changes in mood, acceptance and daily functioning were the most important variables after a psychological programme in the study with chronic pain patients developed by Scott and McCracken (2015). In this study, disability changes present more relevance than pain for the GPES scores suggesting that physiotherapy modalities may have a particular impact on reducing disability perceived by patients. Furthermore, different interventions presented different potential benefits and so the contribution of specific domains to the GPES scores can diverge according to the interventions applied. Looking at the results of this study, they suggest that patients with CLBP undergoing physiotherapy perceive benefits in other domains beyond pain and disability.

Overall, the strength of the associations and the contribution of the independent variables to the GPES scores decreased at the 12-week follow-up. Based on previous studies, these results could be due to recall bias associated to the GPES scale (Kamper et al., 2010b). In fact, different authors have questioned the patients' ability to accurately consider their previous health state when they evaluate the change after long periods of time (Kamper et al., 2010b, 2009). Therefore, the GPES scores at the 12-week follow-up could have been influenced by current pain and disability rather than by the changes that have occurred since the beginning of the intervention. However, this is not the only hypothesis to consider. Over time, patients tend to adapt to the pain condition and adjust their life to minimize its impact. Consequently, other health domains can be valued after the intervention, modifying the importance attributed by patients to pain and disability changes and thus their contribution to the GPES scores. This hypothesis has been argued in other studies (Beaton et al., 2001; Rampakakis et al., 2015).

The effectiveness of the interventions is usually analysed and interpreted considering the absolute values or absolute changes occurring in the various outcome domains. In this study, the analyses using percentage changes showing higher values of correlation coefficients and variance explained the GPES scores. The fact that the percentage changes take into account baseline scores seems to justify these findings (Dworkin et al., 2008; Ostelo et al., 2008). For example, 30 points of pain reduction may represent a complete recovery but also an unsatisfactory change for patients with high baseline levels of pain. Therefore, the analysis of percentage changes should be considered in clinical trials in order to improve the interpretation of the intervention results.

The use of pain intensity and disability to assess the effectiveness of physiotherapy in patients with CLBP has prevailed in recent physiotherapy trials (Pires et al., 2020). These two domains seem to be considered by physiotherapists and researchers as the most important and other outcome domains are rarely used (Pires et al., 2020). The data from this study suggest that the perspective of patients with CLBP and researchers may not be completely aligned and some potential benefits of physiotherapy beyond pain and disability are not being measured. This underrepresentation of patient-centred domains can reduce the validity of the outcome measurement process and should be addressed in future studies. Understanding the perceived benefits by patients with CLBP after physiotherapy can be an important step towards a suitable and valid outcome evaluation.

The knowledge about the importance of pain and disability changes to patients with CLBP undergoing physiotherapy is limited. This study addresses this gap and the findings may have important implications for the way the effectiveness of physiotherapy is measured. Together with the appropriate sample size and variability of recruitment settings (external validity), these were the main strengths of this study. However, some limitations need to be considered. First, a significant proportion of participants failed to attend the 12-week follow-up evaluation. A higher proportion of these participants reported irradiated pain and medication use as well as higher levels of pain intensity at baseline when compared to participants who completed the study. For this reason, the relationship between unavailability to complete the study and worse outcomes after the intervention cannot be excluded. Second, more than $80 \%$ of the participants were women. Although the reasons for this difference are unknown, the findings of this study must be interpreted in the light of this limitation. Third, physiotherapy outcomes (or other intervention) are influenced by multiple contextual factors and other sources of bias (e.g. natural course of LBP; patient's expectations) that cannot be controlled through an observational study design (Testa and Rossettini, 2016). Future studies using more robust analyses (e.g. mediation analysis) and experimental designs must be conducted to confirm our findings (Mansell et al., 2014). Finally, the discussion of the results was based on studies using different samples and interventions than those used in this study. The interpretation of results should be carefully considered and further investigation including patients with CLBP undergoing physiotherapy should be conducted to confirm our findings. 


\section{Conclusion}

This study aimed to clarify the role of pain and disability changes to patients' perceptions about their improvements after a physiotherapy programme. Changes in these domains were significantly related and contributed to explaining a partial proportion of variance in patients' perception of improvement. However, these findings suggested that pain and disability may not be the only potential benefits of physiotherapy perceived by patients with CLBP. The relevance of assessing other outcome domains was reinforced and should be addressed in future studies.

\section{Funders}

DP is supported by Fundação para a Ciência e a Tecnologia - the Portuguese national funding agency for science, research and technology (SFRH/BD/133638/2017).

\section{References}

Airaksinen, O., Brox, J.I., Cedraschi, C., Hildebrandt, J., Klaber-Moffett, J., Kovacs, F., et al., 2006. Chapter 4: European guidelines for the management of chronic nonspecific low back pain. Eur. Spine J. 15.

Beaton, D.E., Tarasuk, V., Katz, J.N., Wright, J.G., Bombardier, C., 2001. "Are you better?" A qualitative study of the meaning of recovery. Arthritis Rheum. 45, 270-279.

Cecchi, F., Pasquini, G., Paperini, A., Boni, R., Castagnoli, C., Pistritto, S., et al., 2014. Predictors of response to exercise therapy for chronic low back pain: result of a prospective study with one year follow-up. Eur. J. Phys. Rehabil. Med. 50 (2), 143-151.

Cook, C., Petersen, S., Donaldson, M., Wilhelm, M., Learman, K., 2017. Does early change predict long-term (6 months) improvements in subjects who receive manual therapy for low back pain? Physiother. Theor. Pract. 33 (9), 716-724.

Cruz, E.B., Fernandes, R., Carnide, F., Vieira, A., Moniz, S., Nunes, F., 2013. Crosscultural adaptation and validation of the Quebec back pain disability scale to European Portuguese language. Spine (Phila Pa 1976) 38, E1491-E1497.

Dworkin, R.H., Turk, D.C., Farrar, J.T., Haythornthwaite, J.A., Jensen, M.P., Katz, N.P., et al., 2005. Core outcome measures for chronic pain clinical trials: IMMPACT recommendations. Pain 113 (1-2), 9-19.

Dworkin, R.H., Turk, D.C., Wyrwich, K.W., Beaton, D., Cleeland, C.S., Farrar, J.T., et al., 2008. Interpreting the clinical importance of treatment outcomes in chronic pain clinical trials: IMMPACT recommendations. J. Pain 9, 105-121.

Evans, R., Bronfort, G., Maiers, M., Schulz, C., Hartvigsen, J., 2014. "I know it's changed": a mixed-methods study of the meaning of Global Perceived Effect in chronic neck pain patients. Eur. Spine J. 23, 888-897.

Farrar, J.T., Young Jr., J.P., LaMoreaux, L., Werth, J.L., Poole, R.M., 2001. Clinical importance of changes in chronic pain intensity measured on an 11-point numerical pain rating scale. Pain 94, 149-158.

Foster, N.E., Anema, J.R., Cherkin, D., Chou, R., Cohen Steven, P., Gross, D.P., et al., 2018. Prevention and treatment of low back pain: evidence, challenges, and promising directions. Lancet (London, England) 391 (10137), 2368-2383.

Freitas, P., Pires, D., Nunes, C., Cruz, E., 2019. Cross-cultural adaptation and psychometric properties of the European Portuguese version of the global perceived effect scale in patients with chronic low back pain. Disabil. Rehabil. 1-7. In press.

Gardner, T., Refshauge, K., McAuley, J., Goodall, S., Hübscher, M., Smith, L., 2015. Patient led goal setting in chronic low back pain-What goals are important to the patient and are they aligned to what we measure? Patient Educ. Counsel. 98 (8), 1035-1038.

Gargon, E., Gurung, B., Medley, N., Altman, D.G., Blazeby, J.M., Clarke, M., et al., 2014. Choosing important health outcomes for comparative effectiveness research: a systematic review. PloS One 9, 12.

Geisser, M.E., Clauw, D.J., Strand, V., Gendreau, R.M., Palmer, R., Williams, D.A., 2010 Contributions of change in clinical status parameters to Patient Global Impression of Change (PGIC) scores among persons with fibromyalgia treated with milnacipran. Pain 149, 373-378.

Gouveia, N., Rodrigues, A., Eusébio, M., Ramiro, S., Machado, P., Canhão, H., et al., 2016. Prevalence and social burden of active chronic low back pain in the adult Portuguese population: results from a national survey. Rheumatol. Int. 36, 183-197.
Green, S.B., 1991. How many subjects does it take to do A regression analysis? Multivariate Behav. Res. 26 (3), 499-510.

Hudson, J.I., Arnold, L.M., Bradley, L.A., Choy, E.H.S., Mease, P.J., Wang, F., et al., 2009. What makes patients with fibromyalgia feel better? Correlations between patient global impression of improvement and changes in clinical symptoms and function: a pooled analysis of 4 randomized placebo-controlled trials of duloxetine. J. Rheumatol. 36 (11), 2517-2522.

Hush, J.M., Refshauge, K., Sullivan, G., De Souza, L., Maher, C.G., McAuley, J.H., 2009. Recovery: what does this mean to patients with low back pain? Arthritis Care Res. 61, 124-131.

Kamper, S.J., Maher, C.G., Herbert, R.D., Hancock, M.J., Hush, J.M., Smeets, R.J., 2010a. How little pain and disability do patients with low back pain have to experience to feel that they have recovered? Eur. Spine J. 19, 1495-1501.

Kamper, S.J., Maher, C.G., Mackay, G., 2009. Global rating of change scales: a review of strengths and weaknesses and considerations for design. J. Man. Manip. Ther. 17, 163-170.

Kamper, S.J., Ostelo, R.W.J.G., Knol, D.L., Maher, C.G., de Vet, H.C.W., Hancock, M.J., 2010b. Global Perceived Effect scales provided reliable assessments of health transition in people with musculoskeletal disorders, but ratings are strongly influenced by current status. J. Clin. Epidemiol. 63.

Kent, P., Keating, J.L., Leboeuf-Yde, C., 2010. Research methods for subgrouping low back pain. BMC Med. Res. Methodol. 10 (62).

Mansell, G., Hill, J.C., Kamper, S.J., Kent, P., Main, C., Van Der Windt, D.A., 2014. How can we design low back pain intervention studies to better explain the effects of treatment? Spine (Phila Pa 1976) 39 (5), 305-310.

Mansell, G., Jordan, K.P., Peat, G.M., Dunn, K.M., Lasserson, D., Kuijpers, T., et al., 2017. Brief pain re-assessment provided more accurate prognosis than baseline information for low-back or shoulder pain. BMC Muscoskel. Disord. 18 (139).

March, L., Smith, E.U.R., Hoy, D.G., Cross, M.J., Sanchez-Riera, L., Blyth, F., et al., 2014. Burden of disability due to musculoskeletal (MSK) disorders. Best Pract. Res. Clin. Rheumatol. 28, 353-366.

Meucci, R.D., Fassa, A.G., Xavier Faria, N.M., 2015. Prevalence of chronic low back pain: systematic review. Rev. Saude Publica 49.

National Institute for Health and Care Excellence, 2016. Low Back Pain and Sciatica in over 16s: Assessment and Management.

Ostelo, R.W.J.G., Deyo, R.A., Stratford, P., Waddell, G., Croft, P., Korff, M Von, et al., 2008. Interpreting change scores for pain and functional status in low back pain. Spine (Phila Pa 1976) 33, 90-94.

Parthan, A., Evans, C.J., Le, K., 2006. Chronic low back pain: epidemiology, economic burden and patient-reported outcomes in the USA. Expert Rev. Pharmacoecon. Outcomes Res. 6, 359-369.

Pires, D., Cruz, E.B., Gomes, L.A., Nunes, C., 2020. How do physical therapists measure treatment outcomes in adults with chronic low back pain? A systematic review. Phys. Ther. https://doi.org/10.1093/ptj/pzaa030 (accept).

Rampakakis, E., Ste-Marie, P.A., Sampalis, J.S., Karellis, A., Shir, Y., Fitzcharles, M.A., 2015. Real-life assessment of the validity of patient global impression of change in fibromyalgia. RMD Open 1, e000146.

Sanderson, T., Morris, M., Calnan, M., Richards, P., Hewlett, S., 2010. What outcomes from pharmacologic treatments are important to people with rheumatoid arthritis? Creating the basis of a patient core set. Arthritis Care Res. 62, 640-646.

Schober, P., Schwarte, L.A., 2018. Correlation coefficients: appropriate use and interpretation. Anesth. Analg. 126 (5), 1763-1768.

Scott, W., McCracken, L.M., 2015. Patients' impression of change following treatment for chronic pain: global, specific, a single dimension, or many? J. Pain 16, 518-526.

Smeets, R.J.E.M., Vlaeyen, J.W.S., Hidding, A., Kester, A.D.M., Van Der Heijden, G.J.M. G., Van Geel, A.C.M., et al., 2006. Active rehabilitation for chronic low back pain: cognitive-behavioral, physical, or both? First direct post-treatment results from a randomized controlled trial [ISRCTN22714229]. BMC Muscoskel. Disord. 7 (5).

Testa, M., Rossettini, G., 2016. Enhance placebo, avoid nocebo: how contextual factors affect physiotherapy outcomes. Man. Ther. 24, 65-74.

Tonidandel, S., LeBreton, J.M., 2011. Relative importance analysis: a useful supplement to regression analysis. J. Bus. Psychol. 26, 1-9.

Verkerk, K., Luijsterburg, P.A.J., Heymans, M.W., Ronchetti, I., Pool-Goudzwaard, A.L., Miedema, H.S., et al., 2013. Prognosis and course of disability in patients with chronic nonspecific low back pain: a 5- and 12-month follow-up cohort study. Phys. Ther. 93 (12), 1603-1614.

Walton, D.M., 2013. What does 'recovery' mean to people with neck pain? Results of a descriptive thematic analysis. Open Orthop. J. 7, 420-427.

Wand, B.M., O'Connell, N.E., 2008. Chronic non-specific low back pain - sub-groups or a single mechanism? BMC Muscoskel. Disord. 9 (11).

Williamson, P.R., Altman, D.G., Blazeby, J.M., Clarke, M., Devane, D., Gargon, E., et al., 2012. Developing core outcome sets for clinical trials: issues to consider. Trials 13. 